\title{
O Estudo de Ondas Periódicas em um Ambiente Virtual de Aprendizagem Colaborativo de Física
}

\author{
Dante Alighieri Alves de Mello ${ }^{12}$, Shirley Takeco Gobara ${ }^{2}$ \\ ${ }^{1}$ Instituto Federal de Mato Grosso do Sul \\ Rua Cinco s/n, Vila Ycaraí, CEP: 79200-000. Aquidauana - MS. \\ ${ }^{2}$ Universidade Federal de Mato Grosso do Sul \\ Cidade Universitária, Caixa Postal 549, CEP: 79070-900. Campo Grande - MS. \\ dante.mello3@gmail.com, stgobaraegmail.com
}

\begin{abstract}
We investigated the occurrence of collaborative learning of "periodic waves" in a Virtual Learning Environment (VLE), called Laboratory Collaborative Learning of Physics (LAFIS). Based on Vygotsky's theory, developed this environment to favor interaction and collaborative participation among students mediated by the teacher. Virtual interactions were analyzed based on Vygotsky's theory associated methodology microgenetic analysis. We found that the quantity and quality of interactions between students and between them and the teacher were essential to the performance of the class in solving the proposed problem.
\end{abstract}

Resumo. Investigamos a ocorrência de aprendizagem colaborativa de "ondas periódicas" em um Ambiente Virtual de Aprendizagem (AVA) denominado Laboratório de Aprendizagem Colaborativo de Física (LAFIS). Com base na teoria de Vygotsky, desenvolvemos esse Ambiente para favorecer a interação e a participação colaborativa entre os estudantes, mediados pelo professor. As interações virtuais foram analisadas com base na teoria de Vygotsky associada à metodologia de análise microgenética. Verificamos que a quantidade e a qualidade das interações entre os estudantes e entre estes e o professor foram essenciais para o bom desempenho da turma na resolução do problema proposto.

\section{Introdução}

Em 2011 iniciamos uma pesquisa cujo objetivo é avaliar as potencialidades de um Ambiente Virtual de Aprendizagem (AVA) denominado LAFIS (Laboratório de Aprendizagem Colaborativa de Física), disponível gratuitamente no endereço http://www.lafis.ufms.br. O LAFIS foi construído com base no AVA denominado LEDVI, sigla para Laboratório Educativo Virtual Interativo [Silva e Gobara 2007]. Assim como no LEDVI, o LAFIS foi projetado para promover a interação e a colaboração virtual entre os estudantes e/ou entre estes e o professor, mediante uma ferramenta de bate-papo (chat), disponível no Ambiente para realizar a atividade proposta.

A diferença entre esses ambientes e aqueles levantados na literatura é que o LAFIS fornece ao professor uma funcionalidade que ainda não existia no LEDVI, que é a possibilidade do professor inserir problemas no Ambiente e alimentar o banco de 
dados ou utilizar os problemas inicialmente disponibilizados por nós ou até mesmo por outros professores.

Em 2013 apresentamos à comunidade científica a concepção e o modo utilização do LAFIS [Mello e Gobara 2013b]. Em cada turma, o professor deverá formar grupos de dois ou três estudantes, que deverão ser orientados para acessar o problema a ser solucionado usando as ferramentas do Ambiente. O professor poderá também verificar as dificuldades de cada aprendiz e mediar as interações para auxiliar na resolução das questões propostas, já que as interações do chat são gravadas em um banco de dados, permitindo, assim, a realização de atividades síncrona ou assincronamente.

Nesse trabalho apresentamos a análise das interações dos estudantes e entre eles e o professor durante a resolução de um problema cadastrado no LAFIS após a realização da etapa piloto da pesquisa.

A "fase piloto" foi fundamentalmente realizada para identificar e corrigir os possíveis erros na apresentação e na programação do Ambiente. A descrição dessa fase encontra-se no artigo Mello e Gobara (2013b). Notamos que a interação entre os estudantes pode não acontecer se o problema proposto no Ambiente não depender do auxílio do colega para ser resolvido. Assim, corrigimos a programação do LAFIS para que determinados elementos fundamentais para a solução do problema ficassem disponíveis para apenas um dos estudantes, com a possibilidade de serem "teletransportados" para o outro laboratório. De acordo com o referencial teórico adotado, a interação é essencial à aprendizagem, portanto, fizemos essa correção na expectativa de aumentar, principalmente, a qualidade das interações entre os estudantes, uma vez que estes teriam que interagir para trocar informações fundamentais à resolução do problema.

O objetivo desse artigo é verificar se as estratégias colaborativas de ensino adotadas, potencializadas pelas interações entre os estudantes, favoreceram a aprendizagem.

\section{Referencial Teórico de Aprendizagem}

O desenvolvimento do LAFIS e a análise das interações dos estudantes nesse Ambiente foram realizados com base na teoria de Vygotsky (2007, 2008). Esse autor destacou que a escola é o espaço social privilegiado para promover o desenvolvimento mental e a internalização de conceitos científicos nos estudantes. Por esse motivo, é fundamental que o professor saiba como acontece o processo de aquisição do conhecimento pelo aprendiz. Para estudar esse processo, Vygotsky (2008, p. 103) buscou responder às seguintes questões: "O que acontece na mente da criança com os conceitos científicos que lhe são ensinados na escola? Qual é a relação entre a assimilação da informação e o desenvolvimento interno de um conceito científico na consciência da criança?"

Para responder essas perguntas, ele, inicialmente, procurou distinguir os chamados conceitos cotidianos e os conceitos científicos. Os conceitos cotidianos dizem respeito às ideias da criança acerca da realidade, desenvolvidas principalmente mediante as interações que ocorrem a partir de suas relações socioculturais estabelecidas nos ambientes familiares e sociais. São conceitos não sistematizados porque originam-se da experiência cotidiana do sujeito, do seu dia-a-dia. Já os conceitos científicos são conceitos formais, geralmente elaborados nas comunidades científicas e educacionais. 
São conceitos de origem abstrata, pois partem de generalizações da realidade. Assim, Vygotsky observou que o desenvolvimento desses dois tipos de conceitos dá-se em direções diferentes, uma vez que a gênese desses conceitos tem origens distintas.

Vygotsky (2008) observou também que a colaboração dos estudantes entre si ou entre eles e o professor é essencial para o desenvolvimento de habilidades e estratégias fundamentais na solução de problemas. Mas, para potencializar a aprendizagem, ressaltou que devemos atuar na chamada Zona de Desenvolvimento Proximal (ZDP) do estudante.

Para explicar o conceito de ZDP, Vygotsky (2007) definiu dois níveis de desenvolvimento humanos: o Nível de Desenvolvimento Real (NDR), que corresponde às funções psíquicas já desenvolvidas e que, ao menos em tese, pode ser medido por testes individuais sem cooperação; e o Nível de Desenvolvimento Potencial (NDP), determinado pela solução de problemas sob a orientação de um adulto ou em colaboração com companheiros que já desenvolveram tais habilidades.

A ZDP é um nível de desenvolvimento intermediário, isto é, no qual o estudante consegue resolver determinados problemas apenas com a mediação de outra pessoa. Futuramente, uma vez que os conceitos associados à resolução desse problema sejam internalizados pelo sujeito, ele conseguirá resolver esse problema independentemente de auxílio. Nesse caso, aconteceu um aumento no nível de desenvolvimento real do aprendiz, o que, para Vygotsky (2007), evidencia que a aprendizagem pode preceder e alavancar o desenvolvimento.

Recentemente, investigamos em artigos, dissertações e teses brasileiras como os pesquisadores vêm propondo o uso das TIC com base na concepção sociointeracionista de Vygotsky para o ensino de física [Mello e Gobara 2013c]. Dentre os 42 trabalhos encontrados, 16 utilizaram como tecnologia os Ambientes Virtuais de Aprendizagem. Em nove desses trabalhos, foram utilizados recursos colaborativos como chat e fóruns, o que se justifica pelo fato da teoria sociointeracionista considerar a interação como condição essencial à aprendizagem. Outros seis trabalhos usaram o AVA para dar suporte a simulações, animações, vídeos, figuras e textos. Nos trabalhos cujo foco de interesse é a colaboração por meio de AVA, a interação proporcionada pelas TIC entre os estudantes e entre eles e o professor foi o fator primordial para o favorecimento da aprendizagem.

Obtivemos também resultados animadores na "fase piloto" de nossa pesquisa, na qual realizamos as primeiras aulas utilizando o LAFIS com os estudantes [Mello e Gobara 2013a]. Propusemos aos estudantes um problema que continha um link para uma simulação do PhET (2014) a respeito de ondas periódicas em uma corda. Os estudantes tinham que configurar a simulação conforme os parâmetros apresentados no enunciado e podiam interagir entre si para identificarem grandezas como comprimento de onda, amplitude, período, frequência e velocidade de propagação da onda. Verificamos que o LAFIS favoreceu a interação colaborativa entre os estudantes investigados, uma vez que $78 \%$ das soluções postadas no chat foram inicialmente debatidas entre os estudantes ou entre estes e o professor. Além disso, os resultados da avaliação escrita, aplicada após a aula no AVA, mostraram que as interações no LAFIS alavancaram o desenvolvimento dos estudantes, pois eles conseguiram resolver problemas que inicialmente só conseguiam resolver com o auxílio dos pares ou do professor. 


\section{Metodologia}

A obtenção dos dados dessa pesquisa aconteceu em quatro etapas, realizadas em seis aulas de 45 minutos cada: (1) primeiramente os estudantes responderam uma avaliação diagnóstica, disponível em http://www.lafis.ufms.br/diagnostico.pdf. Buscamos identificar, nessa avaliação, se determinados conceitos de ondulatória já haviam sido internalizados pelos aprendizes, isto é, se já faziam parte do NDR dos estudantes; (2) após a avaliação diagnóstica, o professor realizou uma aula interativa, na qual foram discutidas as questões solicitadas nessa avaliação; (3) posteriormente, os estudantes acessaram o LAFIS no Laboratório de Informática da escola, buscando, assim, resolverem virtualmente o problema proposto (Quadro 1), contando também com as mediações do professor, quando necessárias; (4) por fim, os estudantes responderam um questionário socioeconômico e também um opinário de avaliação da aula e do Ambiente.

\section{Quadro 1: Problema da "onda periódica" apresentado aos estudantes.}

A figura mostrada no laboratório 2 representa uma onda periódica propagando-se na água (a onda está representada de perfil). Considerando que a velocidade de propagação desta onda é de $4 \mathrm{~m} / \mathrm{s}$ e que cada quadradinho possui $10 \mathrm{~cm}$ de lado, determine:

a) O comprimento de onda.

b) A amplitude da onda.

c) A frequência de oscilação da onda.

d) O período de oscilação do barquinho sobre a onda.

e) o tempo necessário para a onda se deslocar 1 quilômetro $(\mathrm{Km})$.

ATENÇÃO: as equações para solução do problema encontram-se no laboratório 1.

Elementos do Laboratório 1:

$$
\begin{gathered}
v=\frac{\lambda}{T} \\
v=\lambda \cdot f \\
f=\frac{1}{T}
\end{gathered}
$$

Elemento do Laboratório 2:

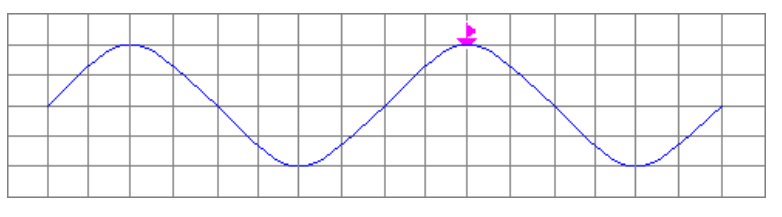

Trata-se de uma pesquisa cuja metodologia é qualitativa, sendo que a análise das interações registradas no chat do LAFIS foi realizada com base na abordagem microgenética, considerada uma sistematização dos princípios metodológicos de Vygotsky [Silva 2013]. Ela possibilita a construção de dados a partir dos recortes de episódios interativos, que permitem uma análise minuciosa das relações intersubjetivas e as condições sociais dos sujeitos investigados. Uma discussão mais aprofundada dessa abordagem encontra-se no artigo de Góes (2000).

Portanto, a partir da observação dos detalhes nos episódios interativos, procuramos identificar as minúcias que indicassem elementos relacionados ao processo de desenvolvimento dos estudantes, pois é na linguagem utilizada pelos estudantes, ao explicarem algo ou ao solucionarem um problema, que encontramos indícios de aprendizagem e desenvolvimento [Silva 2013].

\section{Resultados e Análises}

A aula aconteceu com 20 estudantes do quarto semestre do Curso Técnico Integrado em Edificações, período vespertino, do Instituto Federal de Mato Grosso do 
Sul, na cidade de Aquidauana-MS, no período de Dezembro de 2013 a Janeiro de 2014. O professor pesquisador era também o regente da disciplina.

A Figura 1 mostra a tela disponível para uma estudante alocada no laboratório 2, bem como o problema que os estudantes tiveram acesso nessa aula com o LAFIS. Inicialmente, para "induzir" a interação entre os estudantes, a figura da onda periódica é apresentada apenas no laboratório 2, enquanto as equações necessárias para a resolução encontram-se inicialmente no laboratório 1. Ao clicar sobre a figura é possível enviá-la para o laboratório 2 e vice-versa. O mesmo aplica-se para as equações. Note que, do lado esquerdo da tela, encontra-se o menu de navegação, no meio da tela, apresenta-se o problema da "onda periódica" e, do lado direito, o chat para interação dos estudantes entre si e entre estes e o professor.

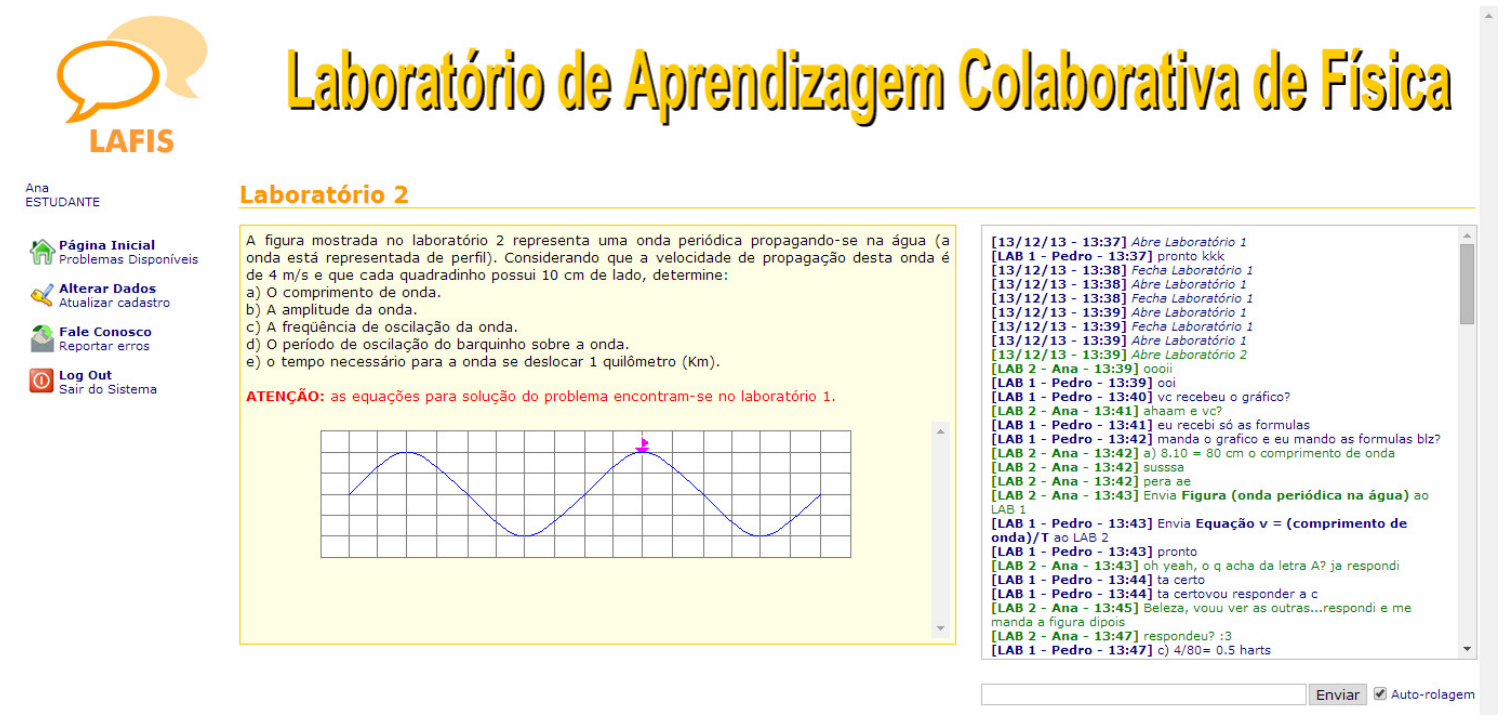

Figura 1: Tela apresentada à estudante alocada no laboratório 2.

Embora seja uma questão de resolução relativamente fácil, a mesma permitiu avaliar o padrão de interação dos estudantes no Ambiente e a compreensão destes quanto aos conceitos de comprimento de onda, amplitude, frequência, período e velocidade de propagação da onda, isto é, suas concepções cotidianas e científicas.

Com o objetivo de explicitar nosso método de análise dos resultados obtidos em sala de aula, transcrevemos abaixo as interações de uma das dez duplas que participaram da resolução desse problema no LAFIS. A escolha dessa dupla para análise em maior profundidade deve-se ao fato de que os estudantes procuraram interagir entre si durante toda a resolução, além de responderem as mediações realizadas pelo professor. Tal qual no Ambiente, as interações estão destacadas nas seguintes cores: azul (Pedro), verde (Ana) e vermelho (Professor). Os nomes desses sujeitos são fictícios.

As respostas de Ana à avaliação diagnóstica indicam que os conceitos fundamentais para a resolução do problema já se encontravam no NDR dessa estudante, visto que, ela conseguiu identificar graficamente os valores do comprimento de onda e da amplitude da onda, além de obter corretamente os valores da frequência e do período da onda por meio das equações fornecidas. Quanto ao estudante Pedro, nada podemos afirmar com relação aos seus conhecimentos prévios, pois o mesmo não respondeu a avaliação diagnóstica. 
Após se cumprimentarem e trocarem, entre si, o gráfico e as equações, os estudantes procuraram interagir para responder as questões propostas:

\section{Episódio 1.}

[13/12/13 - 13:37] Abre Laboratório 1

[13/12/13 - 13:39] Abre Laboratório 2

[LAB 2 - Ana - 13:39] oooii

[LAB 1 - Pedro - 13:39] ooi

[LAB 1 - Pedro - 13:40] vc recebeu o gráfico?

[LAB 2 - Ana - 13:41] ahaam e vc?

[LAB 1 - Pedro - 13:41] eu recebi só as formulas

[LAB 1 - Pedro - 13:42] manda o grafico e eu mando as formulas blz?

[LAB 2 - Ana - 13:42] a) $8.10=80$ cm o comprimento de onda

[LAB 2 - Ana - 13:42] susssa

[LAB 2 - Ana - 13:42] pera ae

[LAB 2 - Ana - 13:43] Envia Figura (onda periódica na água) ao LAB 1

[LAB 1 - Pedro - 13:43] Envia Equação v = (comprimento de onda)/T ao LAB 2

[LAB 1 - Pedro - 13:43] pronto

[LAB 2 - Ana - 13:43] oh yeah, o q acha da letra A? ja respondi

[LAB 1 - Pedro - 13:44] ta certovou responder a c

[LAB 2 - Ana - 13:45] Beleza, vouu ver as outras...respondi e me manda a figura dipois

[LAB 2 - Ana - 13:47] respondeu?:3

Nas interações reproduzidas no episódio 1, Ana identificou corretamente o "comprimento de onda", como já prevíamos pela sua avaliação diagnóstica. Ela também demonstrou uma certa preocupação em saber se Pedro havia obtido a mesma resposta. Pedro, por sua vez, concordou com o valor encontrado pela colega, postando, em seguida, a sua resolução para o cálculo da frequência da onda:

\section{Episódio 2.}

[LAB 1 - Pedro - 13:47] c) 4/80=0.5 harts

[LAB 1 - Pedro - 13:47] Envia Figura (onda periódica na água) ao LAB 2

[LAB 1 - Pedro - 13:48] Envia Equação v = (comprimento de onda)xf ao LAB 2

[LAB 1 - Pedro - 13:48] Envia Equação $f=1 / T$ ao LAB 2

[LAB 1 - Pedro - 13:48] pronto

[LAB 1 - Pedro - 13:51] e) 0.69... h por regra de 3

[LAB 1 - Pedro - 13:52] concorda cm as duas respostas?

As interações do episódio 2 mostram que, para calcular a frequência da onda, Pedro utilizou a equação correta, porém cometeu um erro muito comum: não converteu o valor do comprimento de onda de centímetros para metros. Em seguida, Pedro envia novamente a figura da onda e as equações a Ana, posta sua resposta para o item "e" e pergunta à colega se ela concorda com os seus resultados.

A atitude de Pedro de reenviar a figura e as equações para a colega, conforme solicitação anterior da mesma, reforça a nossa tese de que manter esses elementos em apenas um dos laboratórios, com a possibilidade de serem "teletransportados" é um fator que favorece a interação entre os estudantes. obtidas:

Apresentamos, agora, a discussão entre os estudantes a respeito das respostas

\section{Episódio 3.}

[LAB 2 - Ana - 13:52] ei, ta errado sua B, еи achoo... a minha deu $2 \mathrm{HZ}$;)) a letra B deu $20 \mathrm{~cm}$ -> 2.10 e a $\mathrm{D}$ eu useei a frequência é igual o inverso do período e deu $0,5 \mathrm{~s}$ 
[LAB 1 - Pedro - 13:53] ta mas a b vc fez $\mathrm{cm}$ ?

[LAB 1 - Pedro - 13:53] a q eu fiz foi a c

[LAB 1 - Pedro - 13:54] nao a b

[LAB 1 - Pedro - 13:54] kkkkk

[LAB 2 - Ana - 13:54] eu seei, escrevi errado... mas a minha C deu 2HZ :3

[LAB 2 - Ana - 13:55] kkkkkkkkk

[LAB 1 - Pedro - 13:55] olha

[LAB 1 - Pedro - 13:56] V= comprimento de onda.F

[LAB 1 - Pedro - 13:56] 4=0.8.f

[LAB 1 - Pedro - 13:56] $F=4 / 0.8$

[LAB 1 - Pedro - 13:56] $F=5$

[LAB 1 - Pedro - 13:57] ERREI MSM MAS NAO DEU 2 TBM

[PROFESSOR] Qual a unidade de medida? F $=5$ ?

[LAB 2 - Ana - 13:57] vc errou?' '-'

[LAB 1 - Pedro - 13:57] hz professor

[LAB 1 - Pedro - 13:58] errei pq tinha esquecido de transformar

[LAB 1 - Pedro - 13:58] o comprimento de onda

[LAB 1 - Pedro - 13:58] mas agora acho q ta certo

É interessante notar no Episódio 3 que, após Ana comentar o erro sobre o valor da frequência, Pedro procurou refazer os cálculos, dessa vez postando no chat a resolução passo-a-passo. Assim, ele percebeu que havia se esquecido de fazer a transformação de unidades para o comprimento de onda, chegando à resposta correta de $5 \mathrm{~Hz}$.

Ana, então, analisou as respostas postadas pelo colega e demonstrou ter compreendido que o valor de $5 \mathrm{~Hz}$ seria o correto, como mostra o Episódio 4.

\section{Episódio 4.}

[LAB 2 - Ana - 13:58] eu fiz pelas duas formas e deu resultado diferente

[LAB 2 - Ana - 13:59] vixii eu errei

[LAB 2 - Ana - 13:59] kkkkk sorry

[LAB 2 - Ana - 13:59] esta certooo

[LAB 1 - Pedro - 13:59] kkk de boa

Em seguida, Pedro postou as respostas já obtidas:

\section{Episódio 5.}

[LAB 1 - Pedro - 14:00] entaum fikou assim

[LAB 2 - Ana - 14:00] a minha D deu 0,5s

[LAB 1 - Pedro - 14:00] A) $80 \mathrm{~cm}$

[LAB 1 - Pedro - 14:00] C) $5 \mathrm{hz}$

[LAB 1 - Pedro - 14:01] B) $20 \mathrm{~cm}$

[LAB 2 - Ana - 14:01] D) 0,5s

[LAB 1 - Pedro - 14:01] D) 0.5

[LAB 1 - Pedro - 14:01] s

[LAB 2 - Ana - 14:02] ahaam...concorda com todas? ${ }^{\wedge}$

[LAB 1 - Pedro - 14:02] ah e confere se ta certo ae

[LAB 1 - Pedro - 14:02] sim

[LAB 1 - Pedro - 14:02] ve se a E ta certa

[LAB 2 - Ana - 14:02] ja conferi, pra mim ta certoo :D

[LAB 2 - Ana - 14:03] voou fazer a E agora

[LAB 1 - Pedro - 14:03] blz terminamos

[LAB 2 - Ana - 14:03] ja fez?

[LAB 1 - Pedro - 14:03] sim

[LAB 2 - Ana - 14:03] quantos q deu? 


\title{
[LAB 1 - Pedro - 14:04] 0.69.....
}

[LAB 1 - Pedro - 14:04] horas

Durante as interações descritas no Episódio 5, o professor notou que os estudantes haviam postado duas respostas incorretamente: o item (d), relacionado ao cálculo do período da onda, e o item (e), que solicitava o tempo necessário para a onda percorrer $1 \mathrm{Km}$. Após questionar os estudantes, estes procuraram refazer os seus cálculos:

\section{Episódio 6.}

\author{
[PROFESSOR] Vocês tem certeza que a resposta da letra d é 0,5s? \\ [PROFESSOR] Pedro, esse 0,69 é da letra e? Tem certeza? \\ [LAB 2 - Ana - 14:06] verdade, a D ta errada \\ [LAB 2 - Ana - 14:07] D) 0,2s \\ [PROFESSOR] Pedro, por favor verifique esse valor de 0,2s para o período? \\ [LAB 1 - Pedro - 14:11] E) $250 \mathrm{~s}$ \\ [LAB 1 - Pedro - 14:12] Ana cm ve fez a D? \\ [LAB 1 - Pedro - 14:14] professor a d esta certa \\ [LAB 1 - Pedro - 14:16] D) $0.2 \mathrm{~s}$ \\ [LAB 1 - Pedro - 14:16] $f=1 / T$ \\ [LAB 1 - Pedro - 14:16] 5=1/T \\ [PROFESSOR] É que inicialmente ela postou 0,5... Mas depois chegou no valor de 0,2s \\ [LAB 1 - Pedro - 14:17] 5T=1 \\ [LAB 1 - Pedro - 14:17] $T=1 / 5$ \\ [LAB 1 - Pedro - 14:17] ?T=0.2 s \\ [LAB 1 - Pedro - 14:17] T=0.2s \\ [LAB 2 - Ana - 14:18] é q eu posteei errado professorr...:// \\ [LAB 1 - Pedro - 14:18] passando entaum os resultado \\ [LAB 1 - Pedro - 14:19] A) 80cm B)20cm C) $5 \mathrm{hz}$ D) $0.2 \mathrm{~s} \mathrm{E)} 250 \mathrm{~s}$ \\ [LAB 1 - Pedro - 14:19] certo professor? \\ [PROFESSOR] Certo! Parabéns a vocês! ...
}

Como inicialmente os estudantes encontraram o valor $2 \mathrm{~Hz}$ para a frequência, acabaram por calcular o período como $0,5 \mathrm{~s}$. Postaram também que a resposta do item "e" seria 0,69 horas. Percebendo esses equívocos, como mostrado no Episódio 6, o professor fez uma mediação perguntando se eles tinham certeza desses valores. Assim, os estudantes refletiram sobre suas respostas e conseguiram chegar aos resultados esperados. Devido ao horário escolar, o professor apenas confirmou que as respostas estavam corretas e, como incentivo, elogiou o desempenho do grupo. Porém, se houvesse mais tempo, seria interessante que o professor mantivesse a interação e perguntasse como eles calcularam o item "e", principalmente para identificar as concepções espontâneas de Pedro, que inicialmente postou o valor de $0,69 \mathrm{~h}$. O professor poderia também investigar as concepções espontâneas de Ana, que obteve inicialmente $2 \mathrm{~Hz}$ para a frequência. Assim, seria possível verificar se os conceitos necessários à resolução dessa questão realmente encontravam-se no NDR dos estudantes ou, caso contrário, mediar o grupo no sentido de contribuir para internalizarem tais conceitos.

As análises das respostas desse grupo indicam que as interações, ocorridas durante toda a resolução do problema, entre os estudantes e entre eles e o professor, foram fundamentais e favoreceram a aprendizagem, pois os estudantes precisaram das mediações do professor para solucionar as questões "d" e "e". O fato dos estudantes conseguirem resolver o problema a partir do fornecimento, pelo professor, de algumas 
"pistas", como dizia Vygotsky (2007), sugere que esses conceitos encontravam-se na ZDP dos estudantes.

A Tabela 1 apresenta uma síntese do desempenho geral das dez duplas que participaram da resolução deste problema. Para essa análise, as interações entre os estudantes foram categorizadas em: parcial, para interações em apenas algumas questões; e excelente, para interações colaborativas durante toda a atividade. As interações com o professor foram categorizadas em: insuficiente, nos casos em que não houve interação; boa, para grupos que não fizeram perguntas ao professor, mas que responderam às questões do mestre; e excelente, para grupos que fizeram questionamentos ao professor e que também responderam às questões deste. Já os resultados relacionados à resolução do problema foram categorizados como excelente, para respostas inteiramente corretas.

Tabela 1: Desempenho dos grupos na resolução do problema.

\begin{tabular}{|c|c|c|c|}
\hline Grupo & Interação entre os colegas & Interação com o professor & Resultado \\
\hline 1 & Excelente. & Boa. & Excelente. \\
\hline 2 & Excelente. & Excelente. & Excelente. \\
\hline 3 & $\begin{array}{l}\text { Parcial. Uma estudante estava com } \\
\text { dificuldades, mas não fez perguntas ao } \\
\text { colega. }\end{array}$ & Insuficiente. & Excelente. \\
\hline 4 & $\begin{array}{l}\text { Parcial. Faltou apenas uma discussão do } \\
\text { item "e". }\end{array}$ & Boa. & Excelente. \\
\hline 5 & Excelente. & Excelente. & Excelente. \\
\hline 6 & $\begin{array}{l}\text { Excelente. Uma estudante procurou auxiliar } \\
\text { a colega durante toda a aula. }\end{array}$ & Boa. & Excelente. \\
\hline 7 & Excelente. & Boa. & Excelente. \\
\hline 8 & $\begin{array}{l}\text { Parcial. Discutiram apenas os resultados do } \\
\text { item "c". }\end{array}$ & Boa. & Excelente. \\
\hline 9 & $\begin{array}{l}\text { Parcial. A resolução das questões foi feita } \\
\text { por um estudante de cada vez. }\end{array}$ & Boa. & Excelente. \\
\hline 10 & Excelente. & Insuficiente. & Excelente. \\
\hline
\end{tabular}

Os dados da Tabela 1 mostram que todos os grupos obtiveram êxito na resolução do problema da "onda periódica". Esses resultados são coerentes com o desempenho desses estudantes na avaliação diagnóstica, pois considerando os 18 estudantes que responderam esse questionário, cerca de $81 \%$ das respostas foram respondidas corretamente. Portanto, de acordo com os pressupostos de Vygotsky (2007), esses dados sugerem dois possíveis motivos para justificar o bom desempenho dessa turma na resolução do problema: 1) o fato dos conceitos fundamentais para a resolução do problema já fazerem parte do Nível de Desenvolvimento Real da maioria dos estudantes; 2) a quantidade e qualidade das interações entre os sujeitos e entre estes e o professor, especialmente para aqueles que não tiveram muito sucesso na avaliação diagnóstica.

\section{Considerações Finais}

As análises qualitativas das fontes de dados dessa pesquisa sugerem que o LAFIS, em determinadas condições já enunciadas, favorece a interação colaborativa entre os estudantes, pois a interdependência entre eles no ambiente pode promover 
processos interativos, que podem ser convertidos em ações colaborativas, caso os sujeitos auxiliem um ao outro a resolver os problemas propostos. Dentre os sujeitos investigados, observamos estudantes que receberam auxílio dos seus pares e, assim, conseguiram resolver as questões propostas, bem como casos em que ambos os estudantes de um mesmo grupo não compreendiam ou não sabiam como solucionar o problema, mas que lograram êxito na tarefa ao receberem o auxílio do professor.

Portanto, por suas potencialidades aqui descritas, o LAFIS mostra-se como mais um recurso colaborativo em rede disponível ao professor. Esse Ambiente Virtual de Aprendizagem possibilita que o professor cadastre problemas e forme grupos, de dois ou três estudantes, para resolvê-los. Mesmo que o grupo não solicite o auxílio do professor, ele deve procurar estabelecer a interação, para certificar-se que a resolução realizada pelos estudantes contribuiu para a apropriação dos conhecimentos ou para, simplesmente, constatar que esses conhecimentos já estavam internalizados.

Considerando que em alguns grupos a interação entre os pares foi parcial, principalmente no caso em que apenas um dos estudantes postou as respostas do problema, pretendemos investigar mais profundamente quais ações práticas do professor podem promover a efetiva colaboração entre todos os estudantes.

\section{Agradecimentos}

D. A. A. Mello agradece à CAPES - Brasil pela bolsa de doutorado.

\section{Referências Bibliográficas}

Góes, M. C. R. (2000). A abordagem microgenética na matriz histórico-cultural: uma perspectiva para o estudo da constituição da subjetividade. Cadernos Cedes, 20(50), 9-25.

Mello, D. A. A., e Gobara, S. T. (2013a). Analysis of Interactions in a Virtual Learning Environment Based in Vygotsky's Theory. Creative Education, 10(A), 54-60.

Mello, D. A. A., e Gobara, S. T. (2013b). LAFIS: Um Laboratório Virtual de Aprendizagem Colaborativa de Física. Comunicação apresentada no $9^{\circ}$ Encontro Nacional de Pesquisa em Educação em Ciências. Águas de Lindóia, Brasil.

Mello, D. A. A., e Gobara, S. T. (2013c). O uso das Tecnologias de Informação e Comunicação para o ensino de física com base na teoria sóciointeracionista de Vygotsky. Pôster apresentado no $20^{\circ}$ Simpósio Nacional de Ensino de Física. São Paulo, Brasil.

PhET, Interactive Simulations. (2014). Retirado de http://phet.colorado.edu.

Silva, E. W. F. M., e Gobara, S. T. (2007). LEDVI, Ambiente virtual interativo mediado a distância. Revista Novas Tecnologias na Educação, 5(2).

Silva, H. A. S. (2013). A perspectiva histórico-cultural do desenvolvimento humano: ideias para estudo e investigação do desenvolvimento dos processos cognitivos em ciências. Em R. I. C. Güllich (Org.), Didática das Ciências (pp. 1-25). Curitiba, Brasil: Editora Prismas.

Vygotsky, L. S. (2007). A formação social da mente ( $7^{\mathrm{a}}$ ed.). São Paulo: Martins Fontes.

Vygotsky, L. S. (2008). Pensamento e Linguagem (4 ed.). São Paulo: Martins Fontes. 